\title{
INNOVANDO EN LA EDUCACIÓN PARA LA EMPLEABILIDAD Y EL DESARROLLO DE CARRERA PROFESIONAL EN PUBLICIDAD Y RELACIONES PÚBLICAS. SOCIALOVTT UN CASO DE ESTUDIO.
}

\author{
INNOVATING EDUCATION FOR EMPLOYABILITY AND CAREER \\ DEVELOPMENT IN ADVERTISING AND PUBLIC RELATIONS. \\ SOCIALOVTT, A CASE STUDY.
}

Rosa María Torres Valdés; rosa.torres@ua.es

Alba Santa Soriano; alba.santa@ua.es

Basilio Pueo; basilio@ua.es

Universidad de Alicante

\section{RESUMEN}

Se describe una experiencia conjunta de estudiantes y docentes de Publicidad y Relaciones Públicas para crear una identidad profesional propia mediante el uso eficiente de las redes sociales de internet, cuyo objetivo principal es doble: por un lado, mejorar las posibilidades de empleo del alumno y por otro, promover la innovación y mejora de la calidad docente. Para ello, se genera una acción formativa cuya metodología de Investigación-Acción permite verificar la hipótesis de partida sobre la influencia de la capacidad de manejo de las redes sociales en la relación enseñanza- aprendizaje colaborativos y mejora de oportunidades de inserción laboral. Los resultados iniciales de SocialOVTT animan a pensar en la aplicación de esta experiencia a grupos mayores tales como redes interuniversitarias.

PALABRAS CLAVE: TIC, Relaciones Públicas, Redes sociales, Innovación Educativa, Identidad Digital, Transferencia de Conocimiento, Empleabilidad.

\section{ABSTRACT}

This paper presents a collaborative experience between students and teachers in the knowledge area of advertising and public relations, in order to build a professional identity through the efficient use of social networks and Internet, with two main objectives: on the one hand, to improve the employment possibilities of the students, and by the other hand 
to contribute to innovation performance and improvement of teaching quality. An Action - Research methodology was applied in order to verify the initial hypothesis about the influence that the ability to manage social networks has on the collaborative teaching learning relationship and also on the employment opportunities. Initial results from SocialOVTT encourage thinking in applying this experience to larger groups such as interuniversity networks.

KEYWORDS: ICT, Public Relations, Social Networking, Social Responsibility, Educational Innovation, Digital Identity, Knowledge Transfer, Employability.

\section{INTRODUCCIÓN}

En este artículo se presentan las bases de un estudio que aborda las Tecnologías de la Información y la Comunicación (TIC), la enseñanza - aprendizaje universitarios y la empleabilidad y el desarrollo profesional, con enfoque multidisciplinar a partir de las relaciones públicas (RRPP) y los valores de Responsabilidad Social.

Es conocido que las TIC se han incorporado en todos los ámbitos de la sociedad. Este hecho incide, específicamente, en la relación enseñanza-aprendizaje provocando "una transformación importante, no sólo del lugar en que se aprende sino del cómo se aprende" (Escofet, García González \& Gros Salvat, 2011:1179). En efecto el nuevo entorno en el que nos desenvolvemos está siendo creado por nosotros mismos, gracias a la posibilidad que las TIC nos proporcionan para "ser en la red y crear y participar en comunidades de interés" (Ortega \& Gacitúa, 2008). Por otra parte, hoy día los jóvenes invierten gran parte de su tiempo en los nuevos espacios digitales, situación que invita a los docentes a replantearse la forma de enseñanza, a fin de incrementar la competencia digital propia y de sus alumnos, y así hacer frente a los "desafíos de la enseñanza superior, tanto desde el punto de vista técnico como pedagógico" (Gómez, Roses \& Farias, 2012: 132).

La importancia del tema se evidencia en la abundante literatura, declaraciones y directrices de organizaciones relevantes, sobre la necesidad de alfabetización digital, formación práctica y en responsabilidad social, transferencia de conocimiento y divulgación de los avances científico tecnológicos y enfoques multidisciplinares. Entre otras, cabe citar la siguiente literatura: resumen ejecutivo de la OCDE sobre competencias clave (Rychen \& Salganik, 2004); la propuesta sobre "generación net" y visiones para su educación (Dávila, 2006), la importancia de los "recursos educativos abiertos", definido por la UNESCO como "el material basado en red que se ofrece de forma gratuita y abierta para ser reutilizado en la enseñanza, el aprendizaje y la investigación" (D’Antoni, 2007); el concepto de red de aprendizaje como una comunidad organizada y basada en la comunicación, con objetivo de construir conocimiento (Maldonado, 2008), y la Universidad digital como oportunidad para captación de talento y devolución del conocimiento a la sociedad (Freire y Schuch, 2010). Cada vez cobran más fuerza nuevas culturas de aprendizaje y su incidencia en la educación superior (Escofet, García \& Gros, 2011), el aprendizaje invisible (Cobos \& Moravec, 2011), el mapa de competencias digitales (Ala-Mutka, 2011), ambientes virtuales y formación empresarial (Reinos et. al., 2010), nuevos paradigmas de aprendizaje y las nuevas tecnologías (Esteve \& Gisbert, 2011), Diálogo de saberes (Robles, 2005) y Cuarta Cultura (Lehrer, 2010). 
De hecho, el reciente Informe "Universidad 2020: el papel de las TIC en el nuevo entorno socioeconómicos" (enero de 2012), enfatiza la necesidad de evolucionar de modelos tradicionales de docencia hacia modelos más flexibles, abiertos y participativos.

Además la estrategia Europea 2020, contempla como una de sus iniciativas emblemáticas la Agenda Digital para Europa, cuya misión es "trazar el rumbo que permita maximizar el potencial económico y social de las TICS, y en particular de Internet, como soporte esencial de la actividad económica y social" de los países que integran la Unión Europea, "apostando por la innovación, el crecimiento económico y la mejora de la vida cotidiana de ciudadanos y empresas" (Fundación Telefónica, 2012).

Por su parte, la nueva Ley española 14/2011, de 1 de junio, de la Ciencia, la Tecnología y la Innovación ${ }^{1}$, deja ver una oportunidad para la innovación colaborativa, a la luz de su artículo dos, en el que se destaca como objetivos generales elementos tales como: multidisciplinaridad, interrelación, responsabilidad social y cooperación al desarrollo.

En definitiva, la revisión documental arroja no pocas referencias sobre la relación entre: TIC, transferencia de conocimiento y desarrollo sostenible (entendido como empleo y calidad social), reducción de brechas digitales, comunicación científica de proximidad, redes sociales e identidad digital profesional, innovación educativa y entornos personales de aprendizaje (PLE, por sus siglas en inglés correspondientes a Personal Learning Environment). Estos últimos, se posicionan como una tendencia tecnológica que tendrá un horizonte de implantación de 2 a 3 años (Durall, Gros, Maina, Johnson \& Adams, 2012) y paulatinamente se va instalando en las aulas para facilitar la adquisición de competencias digitales de todos los actores partícipes de la educación y mejorando los procesos de enseñanza-aprendizaje (Gil, 2012), desde una perspectiva centrada en el estímulo de la educación activa y participativa del estudiante (Esteve \& Gisbert, 2011). En esta misma línea, Amine (2009) y Reig (2010) coinciden en definir los PLE como "una colección autodefinida de servicios, herramientas y dispositivos, que ayudan los estudiantes y los docentes a construir Redes Personales de Conocimiento (PKN), poniendo en común nodos de conocimiento tácito (por ejemplo personas) y nodos de conocimiento explícito (por ejemplo información)" (Cabero, Marín \& Infante. 2011:3).

Sin embargo, se aprecia una atomización en el tratamiento de las áreas mencionadas, que si bien han sido estudiadas en profundidad con resultados interesantes no han sido tratadas de modo interdisciplinar. Además, las RRPP como disciplina que promueve el diálogo entre conocimiento y actores quedan fuera de los contenidos de las áreas mencionadas.

Es por ello que de acuerdo con (Cabero, Marín \& Infante. 2011:3) este trabajo desde una aproximación pedagógica-educativa, ha concebido y aplicado los PLE como "sistemas que ayudan a los estudiantes y a los docentes a tomar el control de gestión y de su propio aprendizaje".

El artículo en la Sección 2 describe la metodología investigación-acción seguida y su justificación. Los resultados se presentan en la Sección 3. Finalmente, en la Sección 4 se aborda la discusión y en la Sección 5 se exponen las conclusiones.

${ }^{1}$ http://www.boe.es/boe/dias/2011/06/02/pdfs/BOE-A-2011-9617.pdf 


\section{MATERIALES Y MÉTODOS}

Esta experiencia formativa conlleva una estrategia metodológica de Investigación-Acción. La hipótesis de trabajo se define como sigue: la capacidad de manejo de redes sociales podrían tener influencia relevante en la motivación de alumnos, alumnas y docentes para la enseñanza-aprendizaje colaborativo, así como en la interiorización y aplicación práctica de teorías, modelos y conceptos novedosos, incidiendo todo ello en la mejora de las posibilidades de empleo y autoempleo en su área de conocimiento, en nuestro caso publicidad y relaciones públicas

"La adecuación de la investigación-acción como forma de entender la educación y no sólo de indagar en ella ha sido recogida en la literatura pedagógica" (Romera-Iruela, 2011). En este sentido, la investigación acción o Action Research por su naturaleza comunicacional y de "investigación-intervención-observación participativa" nos ha permitido abordar el trabajo desde el pragmatismo teórico - activo, por ciclos de avance progresivo, tras procesos de reflexión multidisciplinar y democrática.

Para observar los progresos y evaluar el grado de cumplimiento de objetivos, se preparó un cuestionario diseñado ad-hoc pasado a estudiantes y docentes, antes y después de la experiencia SocialOVTT.

La población objeto de estudio ha estado conformada por los 88 alumnos de la asignatura de cuarto curso Teoría y Técnicas de las Relaciones Públicas, por tanto hablamos de un muestreo intencional no probabilístico, para esta experiencia que en sí misma constituye una prueba piloto.

\section{La acción y sus objetivos}

SocialOVTT propone a los alumnos y alumnas el reto de diseñar en equipo un plan de comunicación estratégica para atender a la necesidad de socializar la actividad de un cliente verídico, que en este caso es el Observatorio Virtual de Transferencia de Tecnología (OVTT) de la Universidad de Alicante. A través de esta actividad se estimula al alumno a descubrir un ámbito de conocimiento científico y de futuro desarrollo profesional, nuevo para ellos: RRPP para la divulgación científica de proximidad, explorando nuevas formas de aprenderhaciendo (Carballo, 2006) y construir colaborativamente su identidad digital profesional.

SocialOVTT atiende a los siguientes objetivos didácticos vinculados a competencias:

- Facilitar al alumnado la comprensión e interiorización de conceptos clave, combinando clases presenciales con el uso avanzado de las redes sociales.

- Aplicar dichos conocimientos para lograr los objetivos informativo-comunicativos de la organización cliente (OVTT).

- Estimular la curiosidad, espíritu investigador, pensamiento crítico y trabajo en equipo.

- Explorar nuevos yacimientos de empleo y autoempleo y, con ello, construir su adecuada identidad digital profesional. 


\section{El proceso}

Para alcanzar los objetivos definidos, se parte de un intercambio de expectativas entre los actores participantes y del Briefing aportado por el OVTT, en el que informa de sus necesidades de socialización. A partir de este momento los alumnos y alumnas comienzan a trabajar de forma autónoma y son tutelados a demanda. Las actividades desarrolladas como complemento a la actividad docente formal han sido:

- Construcción inicial y autónoma del Entorno Personal de Aprendizaje (PLE, por sus siglas en inglés de Personal Learning Environment) y selección libre y consensuada de las redes sociales a utilizar por parte de los actores participantes.

- Diagnóstico del nivel y motivación de uso de redes sociales.

- Talleres participativos para el seguimiento del PLE y mejorar las habilidades de activación y utilización profesional de las redes sociales.

- Diseño de la identidad digital de alumnos y alumnas como futuros profesionales.

- Diseño de la identidad digital del docente como actividad de reciclaje profesional.

- Creación colectiva del Cuaderno digital, un espacio de trabajo donde cada equipo comunica sus procesos y comparte las informaciones relacionadas con su cometido en SocialOVTT. Éste se configura en torno al blog de la experiencia (http://SocialOVTT.blogspot.com) como piedra angular, diseñado y gestionado por los propios alumnos y alumnas, y coordinado con las redes sociales empleadas: Twitter, Facebook, YouTube, Slideshare, lector de RSS, marcadores sociales y Google Docs, preferentemente. El contenido producido en el blog es abierto y se difunde en Creative Commons, concretamente bajo licencia abierta CC BY NC ND 3.0.

- Tutorías presenciales y apoyo on-line por parte del docente, y espacio "trucos 2.0".

Los ciclos de avance progresivo pueden visualizarse en la Figura 1, que muestra las fases del proceso y las relaciones entre los actores participantes, así como el rol desempeñado por cada uno en cada etapa. Véase cómo se imparten unos fundamentos de RRPP y alfabetización digital que sirven de base para que el proceso comience su andadura. Asimismo, los docentes guían en el proceso natural de aprendizaje del alumnado, mediando cuando es estrictamente necesario. Por su parte, el alumnado instruye indirectamente al docente, como inmigrante digital, en los contenidos complementarios de los que es experto el docente, provocando en todo este proceso un continuo reciclaje de los métodos y formas de enseñanza. En una segunda fase, el alumnado realiza un proceso de investigación buscando, contactando y entrevistando a profesionales del sector, quienes responden a la petición si así lo consideran oportuno. En este proceso, el docente no influye en ninguno de los dos extremos, ni marcando qué debe decir el alumno ni contactando con el profesional para tratar de dar un matiz institucional a la iniciativa estudiantil. Finalmente, los profesionales y docentes comparten el contenido de las entrevistas, así como el material asociado que se ha ido desarrollando en el proceso. 


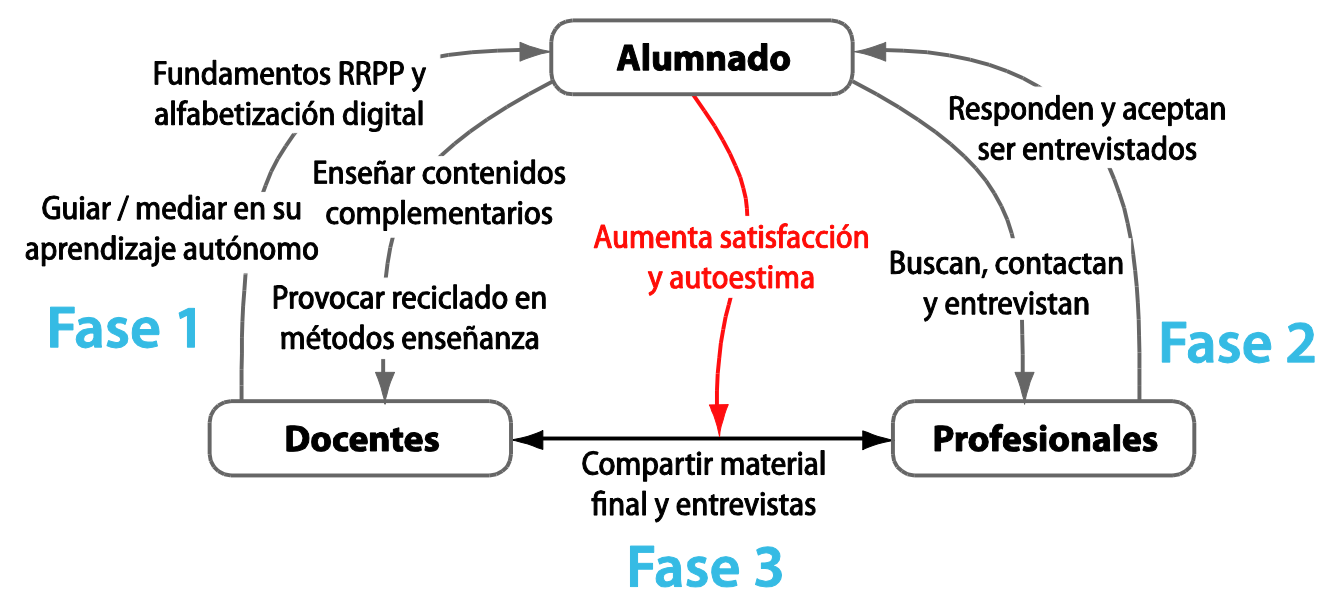

Figura 1: Actores participantes. Fuente: elaboración propia.

En relación con el cuaderno digital, éste es la suma de redes sociales y prácticas abordadas de manera flexible, abierta y participativa desde SocialOVTT y a su vez un instrumento complementario de recogida de información para nuestro estudio. Como muestra la figura 2 , éste contempla tres dimensiones en el uso de las redes sociales por parte de los actores participantes: individual (en rojo), empleadas desde las cuentas personales y aprendiendo a compartir información a través de diversos hashtag; grupal (en verde), gestionadas entre todos los miembros del equipo para una mejora de la eficiencia y coordinación del trabajo en equipo; y de proyecto (en negro), compartiendo un espacio único para el aprendizaje colaborativo y la difusión de la experiencia.

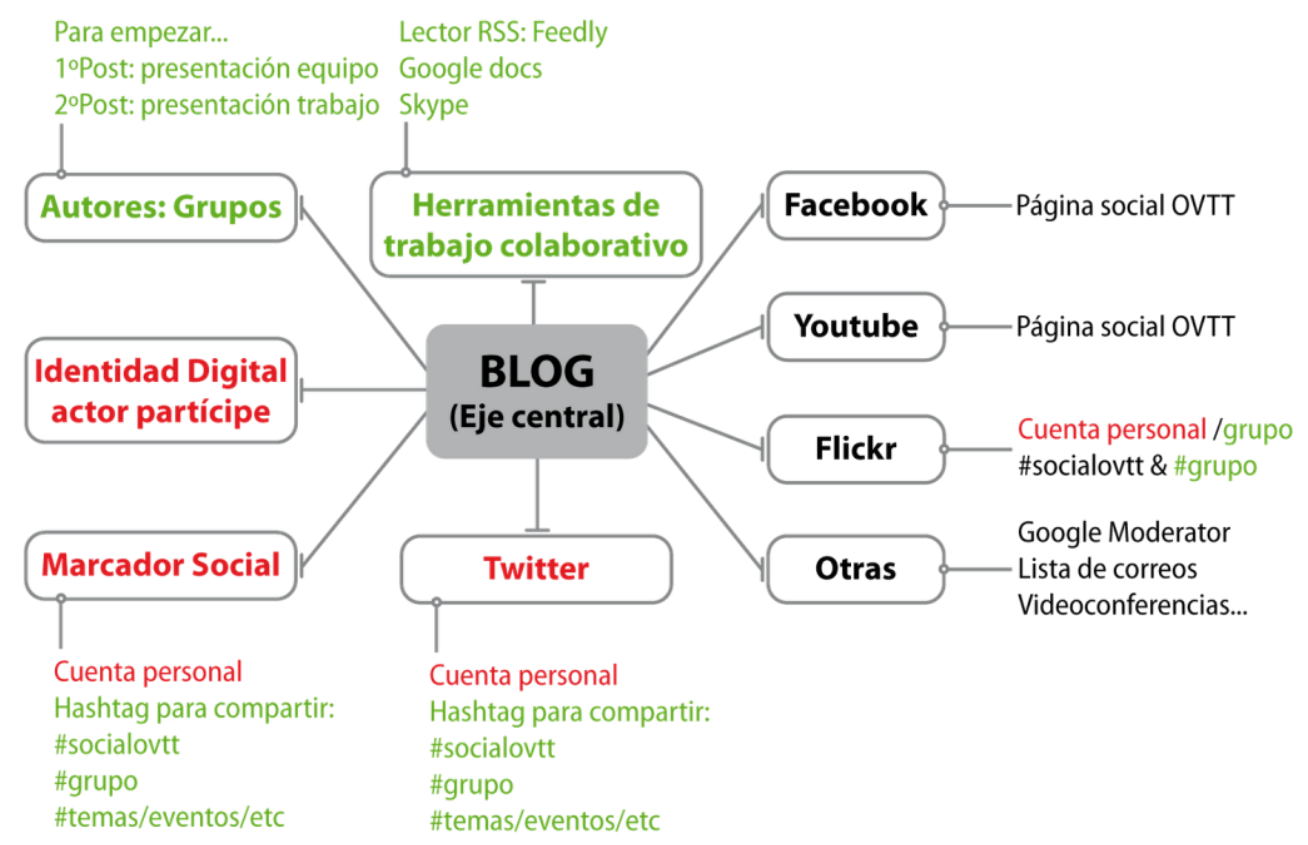

Figura 2: Redes sociales englobadas en el Cuaderno digital. Fuente: elaboración propia.

\section{RESULTADOS}

En este apartado se presentan los resultados de la experiencia SocialOVTT obtenidos a partir del análisis del trabajo de 88 participantes distribuidos en 21 equipos. El primer indicador de 
la aceptación de la iniciativa ha sido la participación: se ha logrado una participación del $73,3 \%$ de alumnos, siendo ésta autónoma, en el sentido de que trabajaban desatendida y voluntariamente, ya que existían otras posibilidades de resolver la asignatura con la misma carga de trabajo que SocialOVTT.

En el blog se han registrado 122 entradas que abordan las temáticas con enfoque interdisciplinar y unas 26.000 visitas recibidas desde distintas localizaciones geográficas, tanto nacionales como internacionales, en un período de seis meses.

Con el fin verificar la hipótesis de partida, y explorar mediante la investigación acción la capacidad de la iniciativa SocialOVTT de dotar a las redes sociales del carácter profesionalizador e integrador, objetivos del proyecto aquí presentado, se diseñaron actividades que permitían obtener resultados tanto cualitativos como cuantitativos. En el primer caso, se invitó a los participantes a expresar de modo abierto y voluntario los comentarios acerca de la experiencia a través de Twitter, enfatizando en sus expectativas y grado de satisfacción tras la experiencia ${ }^{2}$, una vez se había producido la evaluación, para evitar el condicionamiento por la nota final. Los alumnos respondieron positivamente en cuanto a aprendizaje colaborativo, responsabilidad social y aplicación de conceptos a la realidad profesional. Para conocer el grado de incidencia de los distintos conceptos que más han influido en el alumnado, se ha realizado un análisis de frecuencia de las palabras significativas, mediante técnica de saturación del campo del habla, habida cuenta de que la conversación mantenida en Twitter (Strauss y Corbin, 1998: 136). Para ello, una vez filtrados los comentarios de los alumnos y alumnas para extraer los términos significativos, existen unos vocablos cuya frecuencia de aparición es alta, lo cual indica el sentimiento de utilidad generado en ellos y ellas y, sobretodo, y en conexión con los objetivos de motivación, se aprecia el carácter positivo y esperanzador de las palabras más repetidas. Este resultado puede apreciarse en forma de nube de términos de la Figura 3.

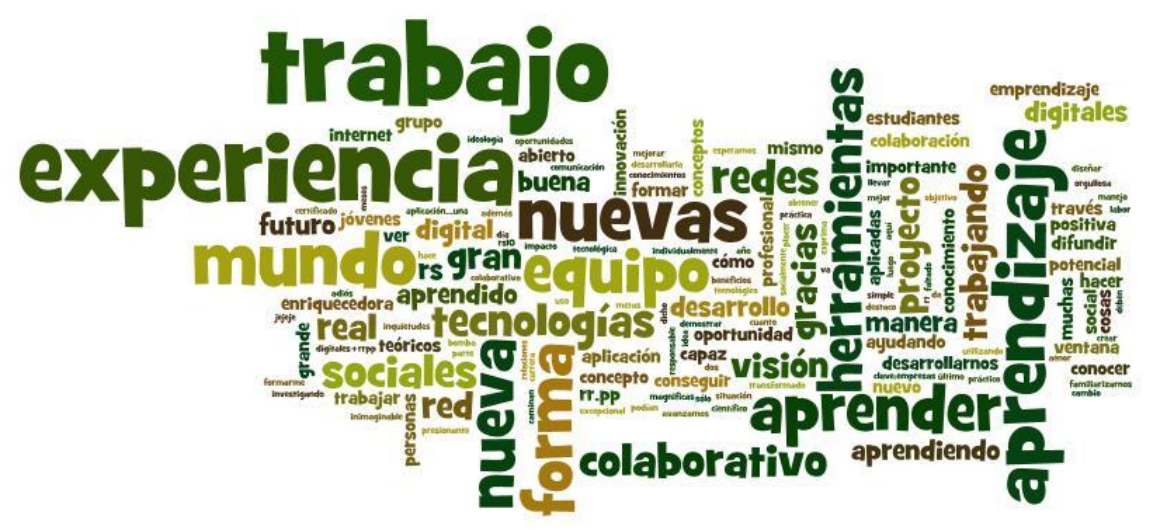

Figura 3: Nube de términos con mayor frecuencia de aparición en los comentarios del alumnado. Fuente: elaboración propia.

Con el fin de analizar los conceptos a los que se refieren las palabras más significativas, se ha realizado un análisis agrupando aquellas que transmiten la misma idea, aunque sea mediante variaciones. Por ejemplo, el concepto "aprender" ha sido expresado como aprender, aprendizaje y aprendido. Atendiendo al conteo de estos términos, los cuatro que más se repiten en los comentarios abiertos de los participantes fueron: trabajo, aprender,

${ }^{2}$ El conjunto de los comentarios es de libre acceso en http://storify.com/OVTT/resume-en-un-tweet-tu-experiencia-de-aprendizaje-e 
nuevo y experiencia. Todos estos términos son muy representativos de la iniciativa llevada a cabo con un colectivo que ha experimentado muchas acciones formativas y que por tanto, es conocedor y exigente en lo que se refiere a su propio aprendizaje (Willingham, 2009).

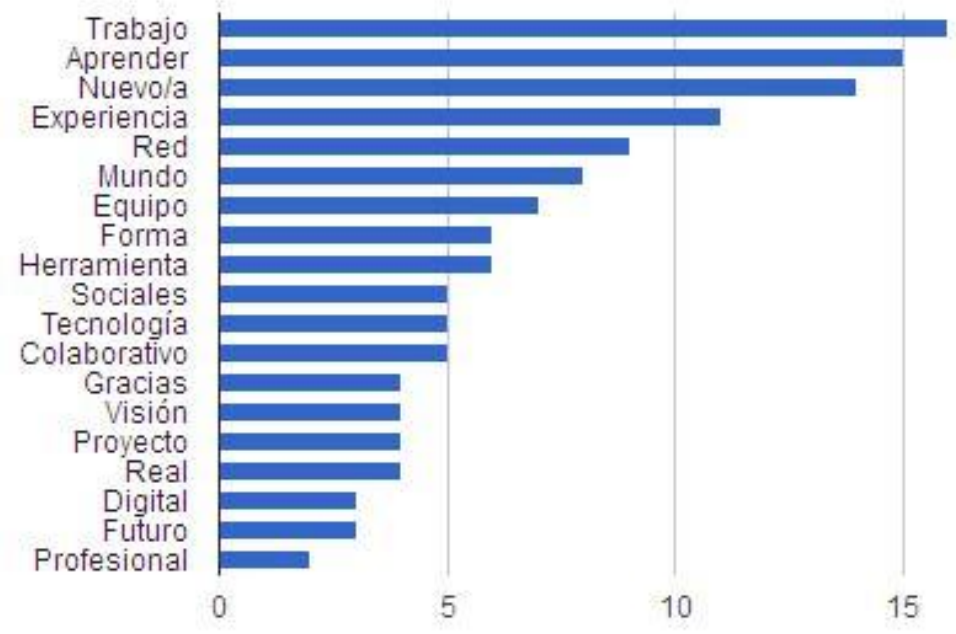

Figura 4: Conceptos con mayor índice de aparición en los comentarios del alumnado. Fuente: elaboración propia.

La segunda herramienta de evaluación de la actividad de carácter cuantitativo consistió en el análisis comparativo de un cuestionario propuesta antes y después de la experiencia. Del estudio de las respuestas se obtienen unos resultados muy interesantes que demuestran, con cifras, el aumento de la destreza digital para la empleabilidad y generación de identidad digital y la sensación en el alumnado de que las herramientas sociales que han venido usando poseen otros fines relacionados con su futuro profesional. Del cuestionario empleado, se han seleccionado unas preguntas claves para el análisis pormenorizado, que se presentan a continuación.

1. ¿Con qué frecuencia navega ahora habitualmente en Internet?

En esta primera cuestión se evaluaba la incidencia en el número bruto de horas que los actores invertían en navegar para detectar si el uso de la herramienta tenía asociado un aumento en el tiempo que se pasaba delante del ordenador. Como se muestra en la Figura 5, los resultados demuestran que el cambio no ha sido significativo ya que la experiencia no parece haber generado adicción a la herramienta, sino que ha conseguido, con el mismo tiempo de uso, ser vista como una herramienta útil, de búsqueda de empleo y establecimiento de identidad digital. El aumentar el número de horas habría sido una consecuencia, si bien lógica, no deseable desde el punto de vista de formación personal. 

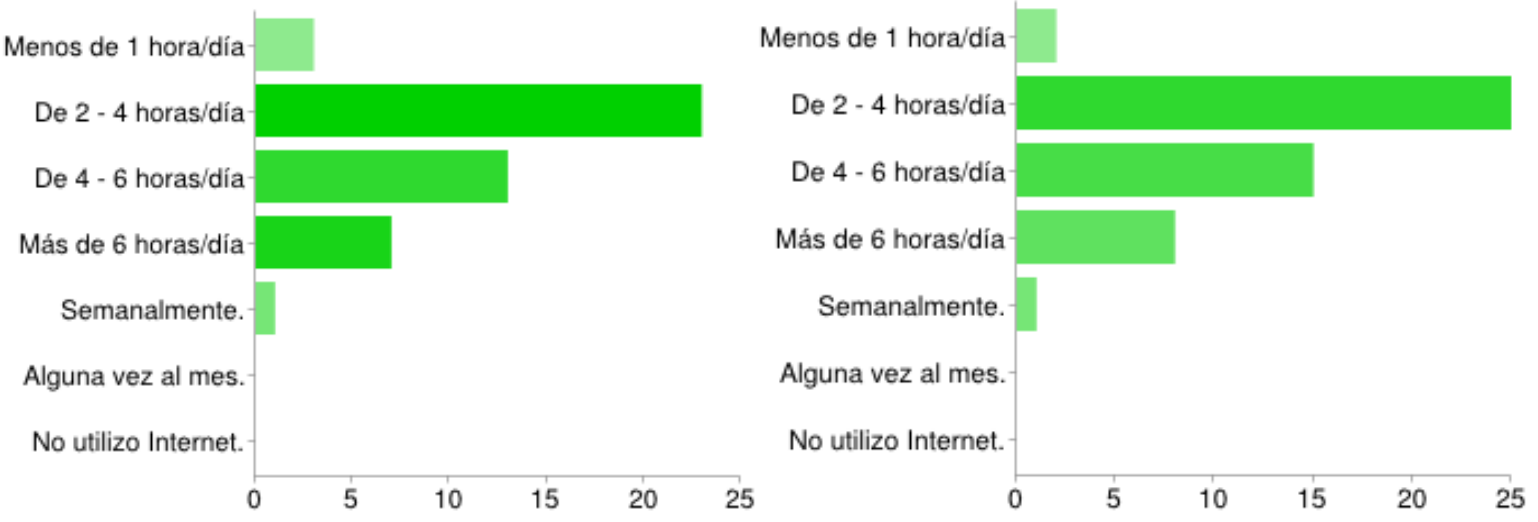

Figura 5: Tiempo de navegación por internet. Izquierda: antes de la actividad, derecha: después. Fuente: elaboración propia.

2. Su acceso y navegación en Internet está más ligado a temas:

En esta cuestión se examinaba las temáticas de la navegación por Internet de forma comparativa para detectar si ha existido un cambio en los intereses de los actores. Como se aprecia en la Figura 6, la herramienta ha provocado un cambio, en algunos casos sustancial, en el patrón de uso de internet a favor de temáticas acordes a los objetivos de la actividad.
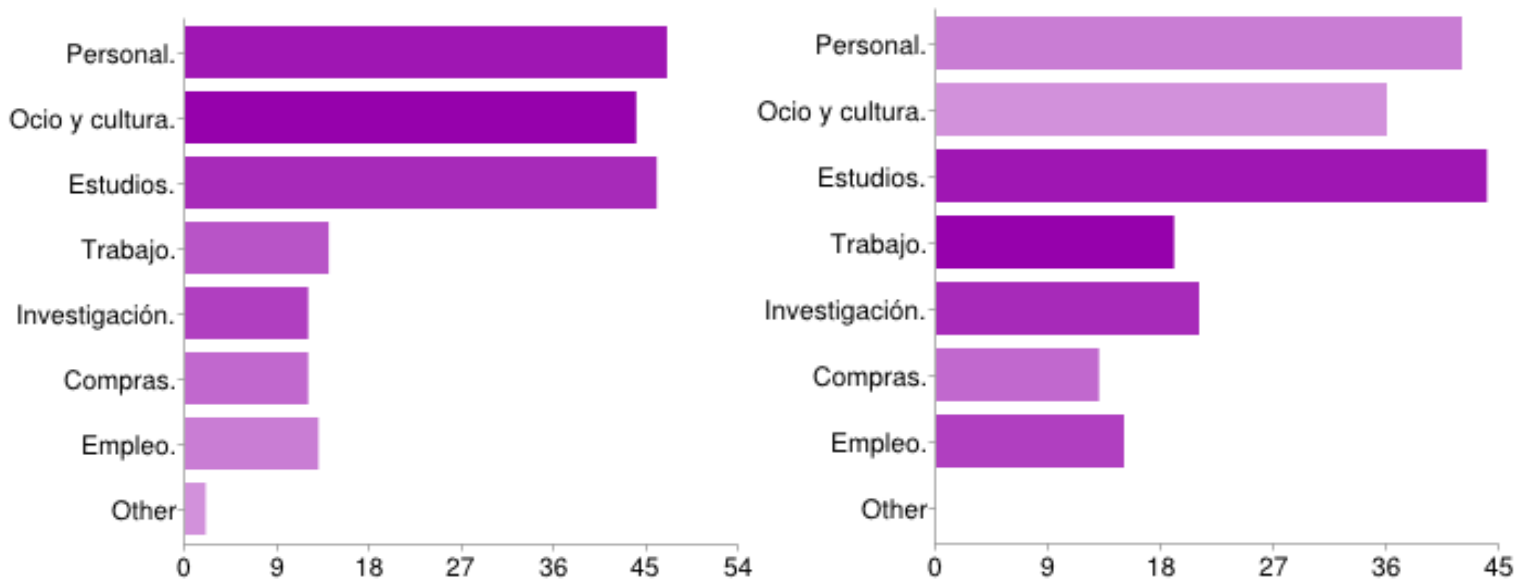

Figura 6: Temática de navegación por internet. Izquierda: antes de la actividad, derecha: después. Fuente: elaboración propia.

Los epígrafes "Estudios" y "Empleo" han tenido un aumento moderado, del $90 \%$ al $94 \%$ en el primer caso, y del $25 \%$ al $32 \%$ en el segundo. Sin embargo, existen dos epígrafes cuyo aumento ha sido muy significativo debido a la dinámica entre el alumnado y el mundo profesional (véase Figura 1): "Investigación" ha aumentado del 24\% al 45\% y "Trabajo" ha aumentado del $27 \%$ al $40 \%$. Este comportamiento es consistente con la investigación que han tenido que realizar para contactar con profesionales con el objetivo de crear las condiciones para obtener un trabajo en el futuro.

3. ¿Qué temáticas de información y contenidos digitales suele consultar en Internet con mayor interés? 
Como continuación de la pregunta anterior, en esta ocasión se exploran los contenidos consultados que no están relacionados directamente con acepciones como trabajo, investigación o empleo para detectar cambios en hábitos derivados de su nuevo carácter investigador/profesional. Como se aprecia en la Figura 7, el alumnado ha cambiado sus preferencias a contenidos más culturales, tecnológicos o científicos y menos de puro entretenimiento.
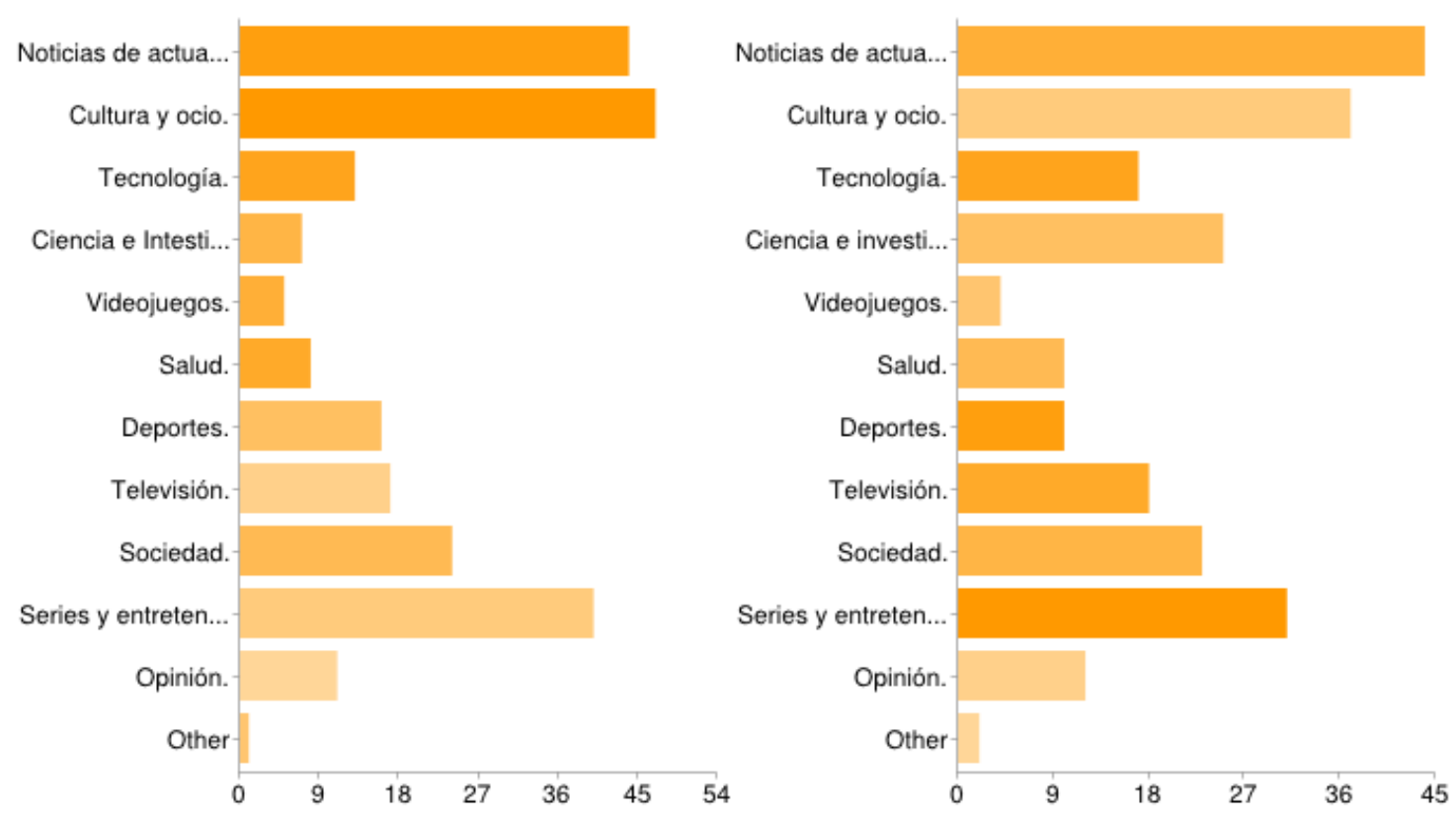

Figura 7: Temáticas y contenidos digitales de consulta en internet. Izquierda: antes de la actividad, derecha: después. Fuente: elaboración propia.

El primer resultado está relacionado con la cultura y el ocio, que ha pasado de tener un 92\% a un $79 \%$, lo cual indica que, si bien estos contenidos son importantes, han dejado un lecho para otros intereses que se describen a continuación. El primero de ellos es el tecnológico, que ha aumentado de $25 \%$ a $36 \%$, cambio significativo para un alumnado no tecnológico como es el caso. El segundo cambio importante es el relacionado con la ciencia e investigación, cuyo incremento del $14 \%$ al $53 \%$ es destacable. Finalmente, como consecuencia de una redistribución de temáticas para un mismo tiempo de consulta, las series y entretenimiento disminuyen de un $78 \%$ a un $66 \%$.

\section{4. ¿Qué medios online utiliza para ello?}

Esta cuestión analiza el modo de obtener información para satisfacer las necesidades, tanto las presentes antes del trabajo, como las adquiridas por la herramienta. Existen cambios específicos relacionados con los objetivos marcados por la herramienta, destacando los siguientes: se ha aumentado de un $8 \%$ a un $26 \%$ el uso de buscadores especializados, así como el uso de gestores RSS, que han pasado de un $14 \%$ a un $36 \%$, datos que demuestran que no eran de conocimiento general antes de comenzar la actividad. Igualmente, es interesante notar que la comunidades virtuales han experimentado un aumento significativo, del $6 \%$ al $32 \%$ en línea con el objetivo transversal de trabajo colaborativo, incluso en escenarios como éste, donde la relación con el profesional podría llevar a iniciativas individuales para obtener un beneficio propio. 
5. Como usuario ¿qué nivel de conocimientos sobre Internet y sus usos considera que tiene ahora mismo?

Esta cuestión evalúa el grado de confianza ganado en el uso de las herramientas con fines de desarrollo personal y, en general, con el uso de Internet. Es una respuesta subjetiva, y como tal, no indica el conocimiento real (mediante una prueba antes/después) sino la confianza ganada en el proceso. Como se aprecia en la Figura 8 , se ha producido un aumento significativo de alumnos y alumnas que se han considerado con mayor nivel tras la iniciativa: ha disminuido en un $18 \%$ la percepción de nivel intermedio para aumentar en un $25 \%$ el nivel avanzado. Nótese también que ningún participante se considera a nivel de iniciado tras realizar la actividad.

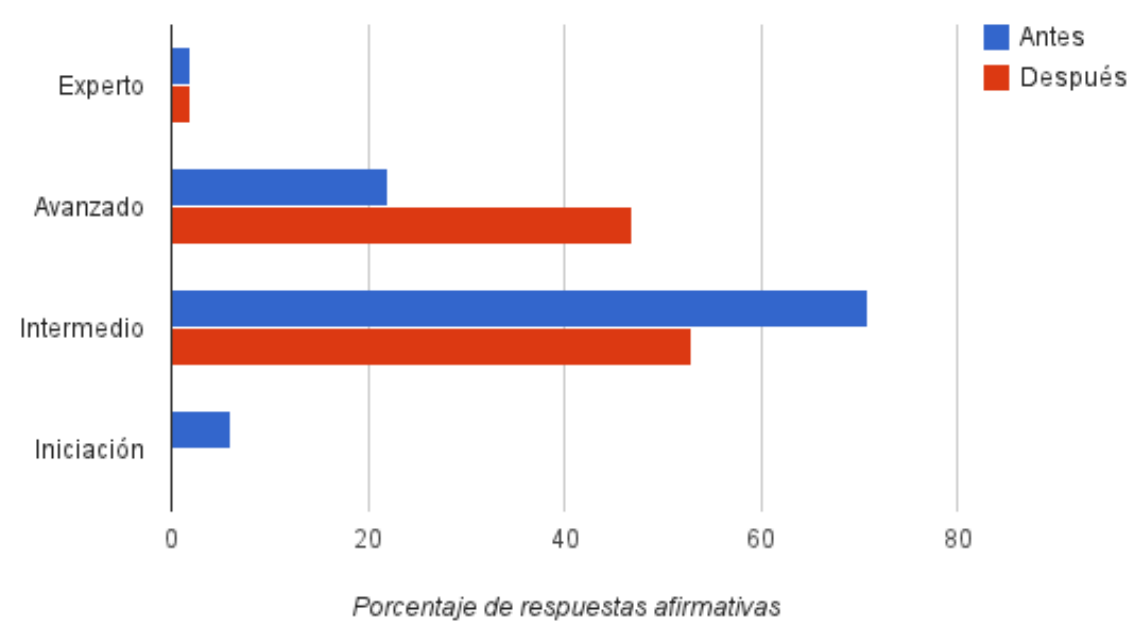

Figura 8: Nivel de conocimiento subjetivo de uso de internet. Fuente: elaboración propia.

\section{6. ¿Conoce qué son los Entornos Personales/Profesionales de Aprendizaje (PLE)?}

El objetivo de esta cuestión era investigar el grado de conocimiento de la herramienta una vez se había practicado por todo el alumnado, siendo el resultado fue especialmente positivo, ya que se produjo un aumento de un $24 \%$ a un $96 \%$.

7. Valore su NIVEL DE SATISFACCIÓN GENERAL con esta experiencia formativa piloto de SOCIALOVTT:

Esta última cuestión, también de carácter subjetivo, resume la satisfacción en términos genéricos de la experiencia. Para evitar el sesgo que se podría producir por la relación académica, la encuesta se pasó una vez los alumnos y alumnas habían sido evaluados. Generalmente este tipo de preguntas suele contestarse con notas bajas cuando la población a la que se le pregunta no está completamente satisfecha y entiende una nota baja como una suerte de castigo ante los organizadores de la acción. Como se muestra en la Figura 9, el nivel de satisfacción ha sido elevado, con el $56 \%$ de los encuestados contestando en el intervalo entre 8 y 10. 


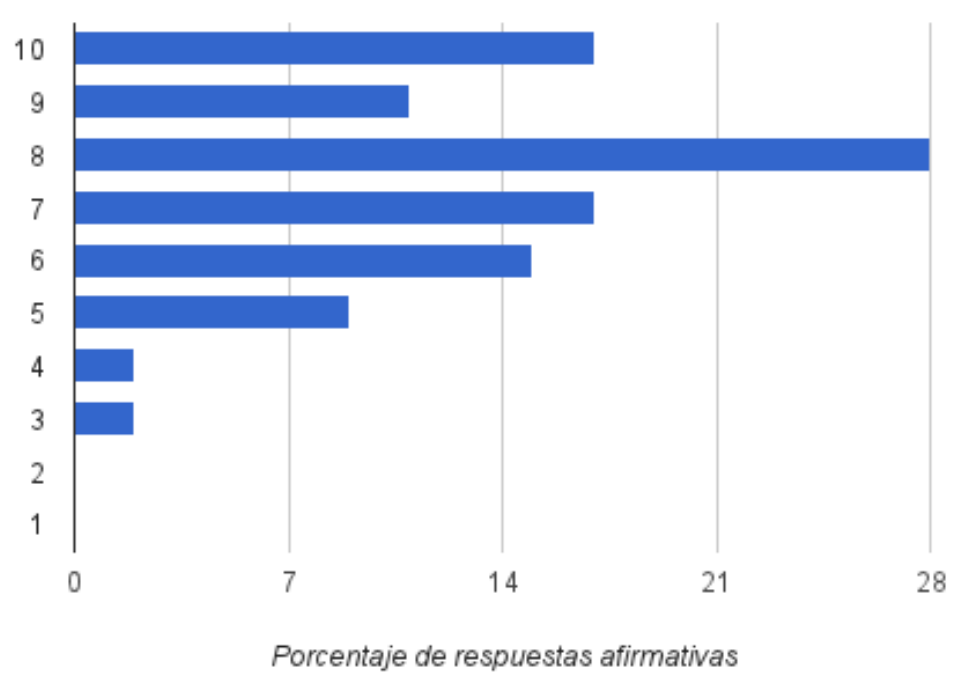

Figura 9: Nivel de satisfacción general tras la realización de la actividad. Fuente: elaboración propia.

\section{DISCUSIÓN}

Autores como Olmedo Estrada (2011), señalan la importancia de la alfabetización científica (que nosotros llamamos comunicación científica de proximidad), en relación con una ciudadanía del S.XXI, informada y participativa. Olmedo señala en su análisis cinco conceptos fundamentales: "comunicación, cognición, responsabilidad social, contextualización, y participación ciudadana en la implantación de políticas públicas relacionadas con incorporaciones tecno-científicas en el devenir cotidiano"; toda una oportunidad para las RRPP en el ámbito científico tecnológico.

Coincidimos con Gómez, Roses y Farias (2011), en que los docentes pueden aprovechar la predisposición de los estudiantes a usar las redes sociales para incorporarlas a la enseñanza, de hecho ésta ha sido la base de nuestra investigación acción, y añadimos que debería aprovecharse también esta disposición, para reeducar en el uso profesional, eficiente, y ético de los recursos tecnológicos. Los casos verídicos son una buena técnica, sin embargo, requiere de gran voluntariedad y esfuerzo extraordinario por parte del docente, para gestionar grupos tan numerosos como en este caso, y el de muchas universidades. Nos encontramos frente a una paradoja: la tecnología como facilitadora de nuevos modos de aprendizaje y la escasez de tiempo por parte del docente cuando el grupo es tan numeroso. Del mismo modo estamos de acuerdo con Giones y Serrat (2010) en que estar en el ciberespacio supone la representación de uno mismo, a través de una identidad digital que se construye mediante la actividad propia y la de los demás en Internet, y precisamente esto es algo sobre lo que socialOVTT, pretendía concienciar a los alumnos, nativos digitales que representan una nueva clase cognitiva (Piscitelli, 2009:37). Por otra parte, nos hallamos en un momento crítico en lo económico, y orientar para "aprender en la incertidumbre" (González \& Tiscar, 2011) parece una necesidad imperiosa. A tenor de los resultados, diríamos que experiencias como SocialOVTT, son de enseñanza-aprendizaje, en la incertidumbre, y de acuerdo con la expresión de González y Tiscar. 
En esta discusión cabe citar el proyecto DeSeCo (Defining and selecting key compentences 2001, o definir y seleccionar competencias fundamentales para la vida 2004) ${ }^{3}$, que sugiere tres funciones para las competencias fundamentales que hacen pertinentes las preguntas expuestas en líneas anteriores. Dichas funciones son:

"Contribuir a resultados valiosos para sociedades e individuos; Ayudar a los individuos a enfrentar importantes demandas en una amplia variedad de contextos y ser relevante tanto para los especialistas como para todos los individuos". Así mismo cabe señalar en el marco del mismo proyecto la declaración conjunta de los ministerios de la OCDE: "El desarrollo sostenible y la cohesión social dependen críticamente de las competencias de toda nuestra población, con competencias que se entiende cubren el conocimiento, las destrezas, las actitudes y los valores". Estas funciones tienen que ver con las palabras clave en torno a las que ha girado SocialOVTT.

\section{CONCLUSIONES}

Los hallazgos de la investigación acción llevada a cabo nos lleva a las siguientes conclusiones:

- En relación con el seguimiento de los ciclos de progreso, se puede afirmar que el enfoque multidisciplinar, ha influido en la apertura de miras, en cuanto a la aplicación práctica de los conocimientos adquiridos durante la carrera en ámbitos profesionales más allá de los tradicionales. La educación como proceso formal institucionalizado también debe orientarse a "la creación de aprendizaje de los procesos de generación de conocimiento" (Martínez, 2008) y la creación de comunidades de práctica, "es un reto necesario a asumir por todos los profesionales de la educación y la formación, dado que a través de ellas se puede, por una parte, transferir y generar nuevo conocimiento y por otra, lograr mejores prácticas en el campo de la investigación y la docencia" (Bozu \& Imbernón, 2009).

- Los resultados indican que la inmensa mayoría de los participantes consideran que la experiencia SocialOVTT ha contribuido a mejorar sus técnicas de estudio, comprender conceptos fundamentales de la asignatura y otras disciplinas, a mejorar la capacidad de investigación, planificación, implementación y evaluación, a trabajar en equipo, compartir en red y crear nuevas posibilidades de empleo y autoempleo. "Las competencias en networking serán útiles para construir una red de fuentes de aprendizaje relevantes" (Reig 2010).

- Mediante el estudio comparativo de las respuestas a encuestas diseñadas para evaluar su competencia y satisfacción, así como consecuencia del análisis de sus comentarios abiertos, podemos concluir que la iniciativa ha sido bien valorada, y los participantes perciben nuevas posibilidades de aplicación de sus conocimientos en nichos de empleo diversos.

- Analizados los registros del Cuaderno Digital, podemos decir que se confirma la hipótesis inicial sobre la eficacia del uso de las herramientas digitales para la motivación hacia el aprendizaje autónomo (Llorens 2009:90), multidisciplinar, y la pérdida de miedo a compartir en la red.

${ }^{3}$ Definición y selección de competencias clave. Resumen ejecutivo. Organización para la Cooperación y el Desarrollo Económico. Página 3 
- Podemos constatar, que una actividad práctica, multidisciplinar y de entrenamiento para el uso avanzado de las redes sociales en ámbito profesional, ha servido de plataforma de lanzamiento de perfiles profesionales del ámbito de la comunicación, posibilitando con ello la visibilidad de los estudiantes ante futuros empleadores. Esta evidencia vino dada con la participación voluntaria de otros profesionales en SocialOVTT, con aportaciones a los contenidos digitales producidos por los actores (entradas en el blog, comentarios, tweets, etc.)

- SocialOVTT se revela como un instrumento de motivación para el reciclaje de docentes y técnicos de I+D en materia de TIC, de modo que la actividad viene a ratificar la hipótesis de partida enunciada.

- Hemos encontrado la posibilidad de formar en estas competencias, con esas funciones, pero se ha hecho desde una asignatura. Quedan pues abiertos interrogantes para futuras investigaciones sobre el tema que nos ha ocupado, preguntas tales como: ¿Es posible mediante el uso eficiente de la tecnología, involucrar más de una asignatura del último curso?, Además de mostrar otros contextos: ¿es posible desarrollar una experiencia tipo SocialOVTT entre facultades distintas dado que hay competencias transversales? ¿Puede involucrarse a más empresarios? ¿Cómo evaluar los impactos en una actividad de tal envergadura, atendiendo a las singularidades de cada especialidad y de cada actor?, ¿Pueden ayudar estas experiencias a mejorar la transferencia de conocimiento y tecnología entre entornos científicos, tecnológicos y productivos?

\section{REFERENCIAS}

ALA-MUTKA, K. (2011). Mapping Digital Competence: Towards a Conceptual Understanding. Technical Note: JRC 67075 - Joint Research Centre - Institute for Prospective Technological Studies.

AMINE, M. "PLE-PKN [Web log post]." 2009. recuperado el 04/05/2013.

BOZU, Z., \& IMBERNÓN MUÑOZ, F. (2009). Creando comunidades de práctica y conocimiento en la universidad: Una experiencia de trabajo entre las universidades de lengua catalana. Revista De Universidad y Sociedad Del Conocimiento, RUSC, 6: 1

CARBALLO, R. (2006). Aprender haciendo. Guía para profesores. Aproximación a los espacios de Aprendizaje basados en la acción, la experiencia y el grupo de trabajo y aplicaciones prácticas. II Encuentro sobre experiencias grupales innovadoras en la docencia universitaria. Universidad Complutense de Madrid.

COBOS, C. \& MORAVEC, J.W. El aprendizaje invisible. Barcelona (España): Laboratori de Mitjans Interactius (UB).

D'ANTONI, S. (2007). Recursos educativos abiertos y contenidos para la educación superior abiertos. Revista De Universidad y Sociedad Del Conocimiento, RUSC, 4(1)

DÁVILA, S. (2006). Generación net: Visiones para su educación. Orbis: Revista De Ciencias Humanas, 1(3), 24-48. 
DURAL, E., GROS, B., MAINA, M., JOHNSON, L. \& ADAMS, S. (2012). Perspectivas tecnológicas: educación superior en Iberoamérica 2012-2017. Austin, Texas: The New Media Consortium.

ESCOFET ROIG, A., GARCÍA GONZÁLEZ, I \& GROS SALVAT, B. (2011). Las nuevas culturas de aprendizaje y su incidencia en la educación superior. Revista Mexicana de Investigación Educativa, 16(51) 1177-1195.

ESTEVE MON, F.M. \& GISBERT CERVERA, M. (2011). El nuevo paradigma de aprendizaje y las nuevas tecnologías. Revista de Docencia Universitaria (REDU), 9: 55-73.

FUNDACIÓN TELEFÓNICA (2012). Universidad 2020: El papel de las TIC en el nuevo entorno socioeconómico. Recuperado el 10 de mayo de 2012 de http://www.fundacion.telefonica.com/es/que hacemos/conocimiento/publicaciones/de $\underline{\text { talle/153 }}$

FREIRE, J. \& SHUCH BRUNET, K. (2010). Políticas y prácticas para la construcción de una Universidad Digital. La Cuestión Universitaria, 6: 85-94.

GIL, M. (2012). Desarrollo de Entornos Personales de Aprendizaje (PLE) para la mejora de la competencia digital. Estudio de caso en una escuela media italiana. Universidad de Burgos.

GUIONES-VALLS, A., \& SERRAT-BRUSTENGA, M. (2010). La gestión de la identidad digital: Una nueva habilidad informacional y digital. BiD: Textos Universitaris De Biblioteconomia i Documentació, 24

GÓMEZ ANGUILAR, M., ROSES CAMPOS, S., \& FARIAS BATLLE, P. (2012). El uso académico de las redes sociales en universitarios. Comunicar: Revista Científica Iberoamericana De Comunicación y Educación, (38), 131-138.

LARA, T. (2011). Aprender en la incertidumbre: Nuevos valores y métodos para formar a los profesionales. Economía Industrial, (381), 27-34.

LEHRER, J. (2010). Proust y la neurociencia. Una visión única de ocho artistas fundamentales de la modernidad. Madrid: Editorial Paidós, 230.

LLORENS LARGO, F. (2009). La tecnología como motor de la innovación educativa. estrategia y política institucional de la universidad de alicante. Arbor: Ciencia, Pensamiento y Cultura, (1), 23-32.

MALDONADO GRANADOS, L.F. \& SERRANO IGLESIAS, E. (2008). Construcción de una red de aprendizaje. Nómadas (Col). 28: 221-222.

MARTÍNEZ GARCÍA, B. (2008). El aprendizaje de la cultura y la cultura de aprender. Convergencia: Revista De Ciencias Sociales, (48), 287-307.

OLMEDO ESTRADA, J. C. (2011). Educación y divulgación de la ciencia: Tendiendo puentes hacia la alfabetización científica. Revista Eureka Sobre Enseñanza y Divulgación De Las Ciencias, 8(2), 137-148. 
ORTEGA SANTAMARÍA, S., \& GACITÚA ARANEDA, J. C. (2008). Espacios interactivos de comunicación y aprendizaje. la construcción de identidades. Revista De Universidad y Sociedad Del Conocimiento, RUSC, 5(2), 5.

PISCITELLI, A. (2009). Nativos digitales. Buenos Aires: Santillana,

REIG HERNÁNDEZ, D. (2010). El futuro de la educación superior, algunas claves. Revista d'Innovació i Recerca en Educació (REIRE), 3: 98-115.

REINOSO LASTRA, J.F., \& MARTíNEZ CÁRDENAS, E.E. (2010). Ambientes virtuales y formación empresarial. Pensamiento y gestión, 28, enero-junio.

ROBLES CASTRILLO, R. (2005). Diálogos entre saberes científicos y artísticos. Colombia: Impresora Feriva S.A. 7

ROMERA-IRUELA, M.J. (2011). La investigación-acción en la formación del profesorado. Revista Española de Documentación Científica, 34, 4, octubre-diciembre, 597-614.

RYCHEN, D. \& SALGANIK, L. (Eds.) (2004) Definir y seleccionar las competencias fundamentales para la vida. México: Fondo de Cultura Económica. (Trad. de Defining and selecting key competencies, Trabajo original publicado en 2001).

STRAUSS, A. y CORBIN, J. (1998). Basics of Qualitative Research: Techniques and Procedures for Developing Grounded Theory. Ed. Sage.

WILLINGHAM, D. (2009). Why Don't Students Like School?: A Cognitive Scientist Answers Questions about How the Mind Works and What It Means for Your Classroom. Ed. John Wiley \& Sons.

\section{Para citar este artículo:}

TORRES, R. M; SANTA, A. \& PUEO, B. (2013). Innovando en la educación para la empleabilidad y el desarrollo de la carrera profesional en publicidad y relaciones pública. SOCIALOVTT un estudio de caso. EDUTEC, Revista Electrónica de Tecnología Educativa, 44. Recuperado el dd/mm/aa de http://edutec.rediris.es/Revelec2/Revelec44/educacion empleabilidad desarrollo carrera profesio nal publicidad relaciones publicas.html 